\title{
Discovery of novel glycerolated quinazolinones from Streptomyces sp. MBT27
}

\author{
Nataliia V. Machushynets ${ }^{1} \cdot$ Changsheng Wu $^{1,2} \cdot$ Somayah S. Elsayed ${ }^{1} \cdot$ Thomas Hankemeier $^{3} \cdot$ Gilles P. van Wezel $^{1} \oplus$
}

Received: 1 December 2018 / Accepted: 19 December 2018 / Published online: 7 February 2019

(c) The Author(s) 2019

\begin{abstract}
Actinobacteria are a major source of novel bioactive natural products. A challenge in the screening of these microorganisms lies in finding the favorable growth conditions for secondary metabolite production and dereplication of known molecules. Here, we report that Streptomyces sp. MBT27 produces 4-quinazolinone alkaloids in response to elevated levels of glycerol, whereby quinazolinones A (1) and B (2) form a new sub-class of this interesting family of natural products. Global Natural Product Social molecular networking (GNPS) resulted in a quinazolinone-related network that included anthranilic acid (3), anthranilamide (4), 4(3H)-quinazolinone (5), and 2,2-dimethyl-1,2-dihydroquinazolin-4(3H)-one (6). Actinomycins D (7) and X2 (8) were also identified in the extracts of Streptomyces sp. MBT27. The induction of quinazolinone production by glycerol combined with biosynthetic insights provide evidence that glycerol is integrated into the chemical scaffold. The unprecedented 1,4-dioxepane ring, that is spiro-fused into the quinazolinone backbone, is most likely formed by intermolecular etherification of two units of glycerol. Our work underlines the importance of varying the growth conditions for the discovery of novel natural products and for understanding their biosynthesis.
\end{abstract}

Keywords 4(3H)-quinazolinones $\cdot$ Molecular networking $\cdot$ Metabolomics $\cdot$ Carbon source

\section{Introduction}

Actinobacteria are a major source of bioactive compounds, producing some two-thirds of all antibiotics as well as molecules with a wide variety of activities such as anticancer, antifungal and immunosuppressant [2, 4]. Traditionally, microbial natural product (NP) discovery has been done via high-throughput screening followed by iterative bioassayguided fractionation and structure elucidation. While such pipelines were extremely successful and delivered a plethora of therapeutic agents, in the modern era the large pharmaceutical companies moved out of NP-discovery programs due to high cost and chemical redundancy [1, 9, 29]. At the same time, the power of genome sequencing brought the recognition that microorganisms harbor a vast and yet untapped biosynthetic potential, and it rapidly became clear that the potential for metabolic diversity of even the beststudied model organisms as producers of natural products had been grossly underestimated $[3,11,19]$. How could these compounds have been missed by the very extensive HT screening campaigns of the twentieth century? The answer is that many of the biosynthetic gene clusters (BGCs) discovered by genome mining are poorly expressed or cryptic 
under laboratory conditions $[22,28]$. A drug-discovery pipeline that is rapidly gaining momentum involves combining genome mining with fluctuating the culturing conditions to achieve differential synthesis of NPs, followed by the metabolic profiling-based identification of the bioactivity of interest [13, 16, 48, 50]. A major challenge thereby lies in finding the appropriate chemical triggers or ecological cues to elicit the production of cryptic antibiotics (recently reviewed in $[34,55,56])$. The use of chemical elicitors is thereby a promising approach $[10,42,56]$.

Manipulation of fermentation conditions for promising producer strains, known as the "one strain many compounds" (OSMAC) approach, is an effective way of enhancing the production of secondary metabolites $[5,33]$. The regulatory networks that control the production of bacterial natural products respond strongly to changes in carbon, nitrogen or phosphate concentration [40, 41]. The reisolation of known metabolites is a major bottleneck in the discovery of new bioactive natural products. A crucial step in this regard is the early identification of already known substances, to concentrate the efforts on the discovery of new ones, a process known as dereplication [15]. Current dereplication strategies include hyphenated techniques, such as LC-MS, LC-NMR, LC-NMR-MS, and LC-SPE-NMR. Bioactivity fingerprinting has also been used to dereplicate natural products based on their biological modes of action [36], while molecular networking is a powerful tool for the visualization and dereplication of natural products [46, 54]. Mass spectrometry-based molecular networking relies on clustering of molecules based on similarities in their MS/MS fragmentation patterns, which depends on the structural features of the ionized molecules. The resulting clusters allow scientists to visually explore the metabolites produced by a given strain under a specific growth condition, allowing rapid dereplication of known compounds by automated spectral library searches, and to visualize their unknown structural analogues [45].

In this work we analyzed the potential of Streptomyces sp. MBT27 as a producer of natural products in response to changes in the carbon source. The strain had previously been identified as a promising producer of NPs [57]. Extensive fluctuations in the secondary metabolite profiles were observed depending on the carbon source used, and statistical methods combined with GNPS molecular networking identified a family of known as well as novel quinazolinone compounds, in response to high concentrations of glycerol. Quinazolinones are heterocyclic compounds with a wide range of medical applications, such as antimicrobial, antiviral, antituberculosis, and as enzyme inhibitors (reviewed in [17, 20, 24]). Combination of MS and NMR methods identified the novel quinazolinones A (1) and B (2), which further expands the chemical space of this rich family of natural products.

\section{Materials and methods}

\section{Bacterial strains and growth conditions}

Streptomyces sp. MBT27 was obtained from the Leiden University strain collection and had previously been isolated from the Qingling Mountains, Shanxi province, China [57]. Cultures were grown in triplicate in $100 \mathrm{~mL}$ Erlenmeyer flasks with $30 \mathrm{~mL}$ of liquid minimal medium (MM; [21]), supplemented with various carbon sources, and inoculated with $10 \mu \mathrm{L}$ of $10^{9} / \mathrm{mL}$ spore suspension. The carbon sources (percentages in $\mathrm{w} / \mathrm{v}$ ) were: $1 \%$ mannitol $+1 \%$ glycerol, $1 \%$ mannitol, $2 \%$ mannitol, $1 \%$ glycerol, $2 \%$ glycerol, $1 \%$ glucose, $2 \%$ glucose, $1 \%$ fructose, $1 \%$ arabinose or $1 \% \mathrm{~N}$-acetylglucosamine (GlcNAc). The cultures were incubated in a rotary shaker at $30{ }^{\circ} \mathrm{C}$ at $220 \mathrm{rpm}$ for 7 days.

\section{General experimental procedures}

NMR spectra were recorded in deuterated methanol $\left(\mathrm{CD}_{3} \mathrm{OD}\right)$ on a Bruker $600 \mathrm{MHz}$ and were referenced using the residual ${ }^{1} \mathrm{H}$ signal of deuterated solvent at $3.30 \mathrm{ppm}[49$, 53]. FT-IR was measured on Perkin-Elmer FT-IR Spectrometer Paragon 1000. UV measurements were performed using a Shimadzu UV mini-1240. Optical rotations were measured on a JASCO P-1010 polarimeter. HPLC purification was performed on Waters preparative HPLC system comprised of 1525 pump, 2707 autosampler, 2998 PDA detector, and Water fraction collector III. The columns used were SunFire $\mathrm{C}_{18}$ column $(5 \mu \mathrm{m}, 100 \AA, 10 \times 250 \mathrm{~mm})$ and SunFire $\mathrm{C}_{18}$ column $(10 \mu \mathrm{m}, 100 \AA, 19 \times 150 \mathrm{~mm})$. TLC was performed using aluminum plates coated with silica gel $60 \mathrm{~F}_{254}$ (Merck). All organic solvents and chemicals were of analytical or LCMS grade, depending on the experiment.

\section{Metabolite profiling}

Following fermentation, culture supernatants were extracted with ethyl acetate (EtOAc) and evaporated under reduced pressure. For LC-ESI-QTOFMS analyses, extracts were dissolved in $\mathrm{MeOH}$ to a final concentration of $1 \mathrm{mg} / \mathrm{mL}$, and $1 \mu \mathrm{L}$ was injected into Waters Acquity UPLC system equipped with Waters Acquity HSS $\mathrm{C}_{18}$ column $(1.8 \mu \mathrm{m}$, $100 \AA, 2.1 \times 100 \mathrm{~mm}$ ), which is coupled to Agilent 6530 QTOF MS equipped with Agilent Jet Stream ESI source (Agilent Technologies, Inc., Palo Alto, CA, USA). For the LC, solvent A was $95 \% \mathrm{H}_{2} \mathrm{O}, 5 \%$ acetonitrile (ACN) and $0.1 \%$ formic acid; solvent $\mathrm{B}$ was $100 \% \mathrm{ACN}$ and $0.1 \%$ formic acid. The gradient used was $2 \% \mathrm{~B}$ for $1 \mathrm{~min}, 2-85 \%$ for $9 \mathrm{~min}, 85-100 \%$ for $1 \mathrm{~min}$, and $100 \%$ for $3 \mathrm{~min}$. The flow rate used was $0.5 \mathrm{~mL} / \mathrm{min}$. As for the MS, the following 
ESI source parameters were used: capillary voltage $3 \mathrm{kV}$, source temperature $325^{\circ} \mathrm{C}$, drying gas flow rate $10 \mathrm{~L} / \mathrm{min}$, and fragmentor $175 \mathrm{~V}$. Full MS spectra were acquired in positive mode in the range of $100-1700 \mathrm{~m} / \mathrm{z}$, in the extended dynamic range mode. Internal reference masses of purine and Agilent HP-921 were continuously delivered to the ESI source through an Agilent 1260 isocratic pump.

Thermo Instruments MS system (LTQ Orbitrap XL, Bremen, Germany) equipped with an electrospray ionization source (ESI) was used for LC-MS/MS analysis. The Waters Acquity UPLC system equipped with Waters Acquity PDA was run using a SunFire Waters $C_{18}$ column $(3.5 \mu \mathrm{m}, 100 \AA$, $4.6 \times 150 \mathrm{~mm})$, at a flow rate of $0.9 \mathrm{~mL} / \mathrm{min}$. Solvent A was $95 \% \mathrm{H}_{2} \mathrm{O}, 5 \%$ acetonitrile (ACN) and $0.1 \%$ formic acid; solvent $\mathrm{B}$ was $100 \% \mathrm{ACN}$ and $0.1 \%$ formic acid. The gradient used was $2 \%$ B for $1 \mathrm{~min}, 2-85 \%$ for $15 \mathrm{~min}, 85-100 \%$ for $3 \mathrm{~min}$, and $100 \%$ for $3 \mathrm{~min}$. As for the MS, the following ESI parameters were used: capillary voltage $5 \mathrm{~V}$, spray voltage $3.5 \mathrm{kV}$, capillary temperature $300^{\circ} \mathrm{C}$, auxiliary gas flow rate 10 arbitrary units, and sheath gas flow rate 50 arbitrary units. Full MS spectra were acquired in the Orbitrap in positive mode at a mass range of $100-2000 \mathrm{~m} / z$, and FT resolution of 30,000. Data-dependent $\mathrm{MS}^{2}$ spectra were acquired in the ion trap for the three most intense ions using collision induced dissociation (CID). The resulting chemical data were compared with those in SciFinder and Antibase [25].

\section{Large-scale fermentation and isolation of metabolites (1) and (2)}

For large-scale fermentation, Streptomyces sp. MBT27 was grown in eight $2 \mathrm{~L}$ Erlenmeyer flasks, each containing $500 \mathrm{~mL}$ liquid MM supplemented with $2 \%$ w/v glycerol at
$30{ }^{\circ} \mathrm{C}$ for 7 days. The metabolites were extracted from the spent media of culture filtrates using EtOAc, and the solvent was subsequently evaporated under reduced pressure at $40{ }^{\circ} \mathrm{C}$. The crude extract $(1.4 \mathrm{~g})$ was adsorbed onto $1.4 \mathrm{~g}$ silica gel (pore size $60 \AA$, 70-230 mesh, Sigma Aldrich), and loaded on silica column, which was eluted using gradient mixtures of $n$-hexane, acetone, and $\mathrm{MeOH}$. The fractions eluted with acetone $100 \%$ were combined, reconstituted in $\mathrm{MeOH}$, and injected into the preparative SunFire column $(19 \times 150 \mathrm{~mm})$, which was eluted with $\mathrm{H}_{2} \mathrm{O}: \mathrm{MeOH}$ gradient of $7-100 \%$ in $30 \mathrm{~min}$, at a flow rate of $15 \mathrm{~mL} / \mathrm{min}$, to yield five fractions. Quinazolinones A and B were further purified on the semi-preparative SunFire column $(10 \times 250 \mathrm{~mm})$, run at $3 \mathrm{~mL} / \mathrm{min}$. Fraction 2 was eluted using $\mathrm{H}_{2} \mathrm{O}: \mathrm{MeOH}$ gradient of $20-40 \%$ in 20 min, to yield Quinazolinone A $(1,1 \mathrm{mg})$. On the other hand, Fraction 3 was eluted using $\mathrm{H}_{2} \mathrm{O}: \mathrm{MeOH}$ gradient of $30-40 \%$ in 20 min, to yield Quinazolinone B $(2,1.5 \mathrm{mg})$.

\section{Quinazolinone A (1)}

Colorless, amorphous powder; $[\alpha]_{\mathrm{D}}^{20} 2.3$ (c $\left.0.1, \mathrm{MeOH}\right)$; $\mathrm{UV}(\mathrm{MeOH}) \lambda_{\max }(\log \varepsilon) 224$ (4.09), $322(2.98) \mathrm{nm} ; \mathrm{IR}$ $\nu_{\max } 3334,2922,1652,1475,1052 \mathrm{~cm}^{-1} ;{ }^{1} \mathrm{H}$ and ${ }^{13} \mathrm{C}$ NMR data, see Table 1; HRESIMS (positive mode) $\mathrm{m} / \mathrm{z} 281.1130$ $[\mathrm{M}+\mathrm{H}]^{+}$(calcd. for $\mathrm{C}_{13} \mathrm{H}_{17} \mathrm{~N}_{2} \mathrm{O}_{5}, 281.1132$ ).

\section{Quinazolinone B (2)}

colorless, amorphous powder, UV (MeOH) $\lambda_{\max }(\log$ ع) 226 (4.18), $347(3.03) \mathrm{nm}$; IR $\nu_{\max } 3350,2950,1649$, 1526, 1049, $751 \mathrm{~cm}^{-1} ;{ }^{1} \mathrm{H}$ and ${ }^{13} \mathrm{C}$ NMR data, see Table 1;
Table $1{ }^{1} \mathrm{H}$ and ${ }^{13} \mathrm{C}$ NMR data for compounds 1 and 2

\begin{tabular}{|c|c|c|c|c|}
\hline \multirow[t]{2}{*}{ NO. } & \multicolumn{2}{|l|}{1} & \multicolumn{2}{|l|}{2} \\
\hline & $\delta_{C}$ & $\delta_{H}($ mult., $J$ in $\mathrm{Hz}$ ) & $\delta_{C}$ & $\delta_{H}$ (mult., $J$ in $\mathrm{Hz}$ ) \\
\hline 2 & 79.0 & & 73.3 & \\
\hline 4 & 166.1 & & 166.7 & \\
\hline $4 \mathrm{a}$ & 121.8 & & 114.5 & \\
\hline 5 & 128.8 & $7.88(\mathrm{dd}, J=7.8,1.2)$ & 128.5 & $7.66(\mathrm{dd}, J=7.8,1.2)$ \\
\hline 6 & 123.0 & $7.07(\mathrm{t}, J=7.8)$ & 118.5 & $6.69(\mathrm{td}, J=7.8,1.2)$ \\
\hline 7 & 134.6 & $7.46(\mathrm{td}, J=7.8,1.2)$ & 135.3 & $7.26(\mathrm{td}, J=7.8,1.2)$ \\
\hline 8 & 122.3 & $7.23(\mathrm{brd}, J=7.8)$ & 115.8 & $6.73(\mathrm{brd}, J=7.8)$ \\
\hline $8 \mathrm{a}$ & 143.7 & & 148.6 & \\
\hline $1^{\prime}$ & 73.9 & $4.43(\mathrm{~d}, J=9.0) ; 4.24(\mathrm{~d}, J=9.0)$ & 64.5 & $3.67(\mathrm{~d}, J=10.8) ; 3.62(\mathrm{~d}, J=10.8)$ \\
\hline $2^{\prime}$ & 64.6 & $3.59(\mathrm{~d}, J=11.4) ; 3.47(\mathrm{~d}, J=11.4)$ & 64.5 & $3.67(\mathrm{~d}, J=10.8) ; 3.62(\mathrm{~d}, J=10.8)$ \\
\hline $1^{\prime \prime}$ & 65.7 & $3.86(\mathrm{~d}, J=12.6) ; 3.80(\mathrm{~d}, J=12.6)$ & & \\
\hline $2^{\prime \prime}$ & 102.9 & & & \\
\hline $3^{\prime \prime}$ & 61.9 & $3.66(\mathrm{~d}, J=3.0)$ & & \\
\hline
\end{tabular}

1 and 2 were recorded in $\mathrm{CD}_{3} \mathrm{OD}$, at $298 \mathrm{~K}$. All chemical shift assignments were done on the basis of 1D and 2D NMR techniques 
HRESIMS (positive mode) $\mathrm{m} / \mathrm{z} 209.0915[\mathrm{M}+\mathrm{H}]^{+}$(calcd. for $\mathrm{C}_{10} \mathrm{H}_{13} \mathrm{~N}_{2} \mathrm{O}_{3}, 209.0921$ ).

\section{Computation of mass spectral networks}

MS/MS raw data were converted to a 32-bit mzXML file using MSConvert (ProteoWizard) [6] and spectral networks were assembled using Global Natural Product Social molecular networking (GNPS) (https://gnps.ucsd.edu) as described [45]. For both parent and MS/MS fragment ions, the mass tolerance was set to $0.5 \mathrm{Da}$, while the minimum cosine score was set to 0.7 . The data were clustered using MSCluster with a minimum cluster size of three spectra. The spectra in the network were also searched against GNPS spectral libraries. A minimum score of 0.5 was set for spectral library search, with at least two fragment peaks matching. Cytoscape 3.5.1 was used for visualization of the generated molecular networks [38]. The edge thickness was set to represent the cosine score, with thicker lines indicating higher similarity between nodes. LC-MS/ MS data were deposited in the MassIVE Public GNPS data set (MSV000082988). The molecular networking job in GNPS can be found at https://gnps.ucsd.edu/ProteoSAFe/ status.jsp?task $=8 \mathrm{fd} 1 \mathrm{fcfa} 0 \mathrm{ff} 744 \mathrm{a} 9808 \mathrm{e} 80 \mathrm{bc} 7 \mathrm{be} 12115$. The annotated MS/MS spectra were deposited in the GNPS spectral library for quinazolinone A (CCMSLIB00004684355), and B (CCMSLIB00004684354).

\section{Statistical analysis}

Prior to statistical analysis, mzXML files were imported into Mzmine 2.31 [30] for data processing. Mass ion peaks were detected using the exact mass algorithm with a noise level set to $1.0 \times 10^{4}$. Afterwards, chromatograms were built for the detected masses with a minimum time span of $0.05 \mathrm{~min}$, $\mathrm{m} / \mathrm{z}$ tolerance of $0.001 \mathrm{~m} / \mathrm{z}$ and minimum height of $1.0 \times 10^{4}$. Chromatogram deconvolution was then performed using local minimum search algorithm (search minimum in RT range $0.1 \mathrm{~min}$, chromatographic threshold $90 \%$, minimum relative height $1 \%$, minimum absolute height $1.0 \times 10^{4}$, minimum ratio of peak top/edge two and peak duration range $0.05-3 \mathrm{~min}$ ). In the generated peak lists, isotopes were identified using isotopic peaks grouper $(\mathrm{m} / \mathrm{z}$ tolerance $0.001 \mathrm{~m} / \mathrm{z}$ and retention time tolerance $0.1 \mathrm{~min}$ ), and variations in retention time were reduced using retention time normalizer $(\mathrm{m} / \mathrm{z}$ tolerance $0.001 \mathrm{~m} / \mathrm{z}$ and retention time tolerance $1 \mathrm{~min}$ ). All the peak lists were subsequently aligned using join aligner $(\mathrm{m} / \mathrm{z}$ tolerance $0.001 \mathrm{~m} / \mathrm{z}, \mathrm{m} / \mathrm{z}$ weight 20 , retention time tolerance $0.1 \mathrm{~min}$, and retention time weight 20 ), and missing peaks were detected through gap filling using peak finder (intensity tolerance $1.0 \%, \mathrm{~m} / \mathrm{z}$ tolerance $0.001 \mathrm{~m} / \mathrm{z}$, and retention time tolerance of $0.2 \mathrm{~min}$ ). Finally, the aligned peak list was exported as a comma-separated file for statistical analysis.

Statistical analysis was performed using MetaboAnalyst [7], where $\log$ transformation and pareto scaling was initially applied to the data. A heat map of all detected masses, among the different growth conditions, was generated in MetaboAnalyst, to which additional hierarchical clustering analysis (HCA) was performed using Euclidean distance measure and Ward clustering algorithm. Student's $t$-tests with multiple testing correction (Benjamini-Hochberg-false discovery rate or FDR) were used to determine significant differences in the intensities of the metabolites, under two different growth conditions. The thresholds set for statistically significant differences were a fold change $\geq 4$, together with FDR corrected $p$ value $\leq 0.05$. Based on these criteria, a volcano plot was generated. To identify the difference in intensity of a single mass feature among multiple growth conditions, one-way ANOVA was performed, followed by a post hoc Tukey's honest significant difference (HSD) test.

\section{Results and discussion}

\section{The influence of carbon sources on secondary metabolite production}

Previous screening of our in-house actinomycete collection, obtained from remote mountain soils, showed it is a promising source of new bioactive compounds [57]. Under specific growth conditions, these isolates exert potent inhibitory activity against the so-called ESKAPE pathogens [31] Enterococcus faecium, Staphylococcus aureus, Klebsiella pneumoniae, Acinetobacter baumannii, Pseudomonas aeruginosa and Enterobacter spp. In the current study, the potential of one of the Streptomyces strains in our collection, namely Streptomyces sp. MBT27, was analyzed to study the effect of carbon sources on its metabolite profile. Traditional approaches, such as changes in fermentation conditions, are known to induce significant changes in the microbial metabolome. Culture medium components, and particularly the carbon source, have major effects on the production of secondary metabolites $[5,26,35,43]$. The differential production of small molecules is ideal for metabolomics studies, whereby metabolic variations are correlated statistically to bioactivity, thus facilitating the identification of the bioactive molecule of interest $[48,51,52]$. To establish the potential of Streptomyces sp. MBT27, the strain was grown in liquid minimal media containing different carbon sources, namely (percentages in w/v): $1 \%$ of both mannitol and glycerol, $1 \%$ mannitol, $2 \%$ mannitol, $1 \%$ glycerol, $2 \%$ glycerol, $1 \%$ glucose, $2 \%$ glucose, $1 \%$ fructose, $1 \%$ arabinose, or $1 \%$ $\mathrm{N}$-acetylglucosamine (GlcNAc). The latter is an elicitor 
of antibiotic production, via metabolic interference with the global nutrient sensory network controlled by DasR $[32,39]$. Phosphate buffer was omitted from the MM as it repressed the production of secondary metabolites by Streptomyces sp. MBT27 (data not shown). To identify the secondary metabolites in the cultures, supernatants were extracted with EtOAc, and the resulting crude extracts subjected to LC-MS analysis. Using the LC-MS data, a heat map with added hierarchical clustering was generated, to visualize the production of different metabolites under different culture conditions (Fig. 1). Hierarchical clustering analysis of the LC-MS data allows effective comparative analysis of metabolomics data, and the heat map revealed major differences in the metabolic profiles of Streptomyces sp. MBT27, whereby different groups of metabolites were enhanced depending on the carbon source used. Interestingly, not only the type of carbon source, but especially also the concentration resulted in large changes in the metabolic profiles. Doubling the concentration of either

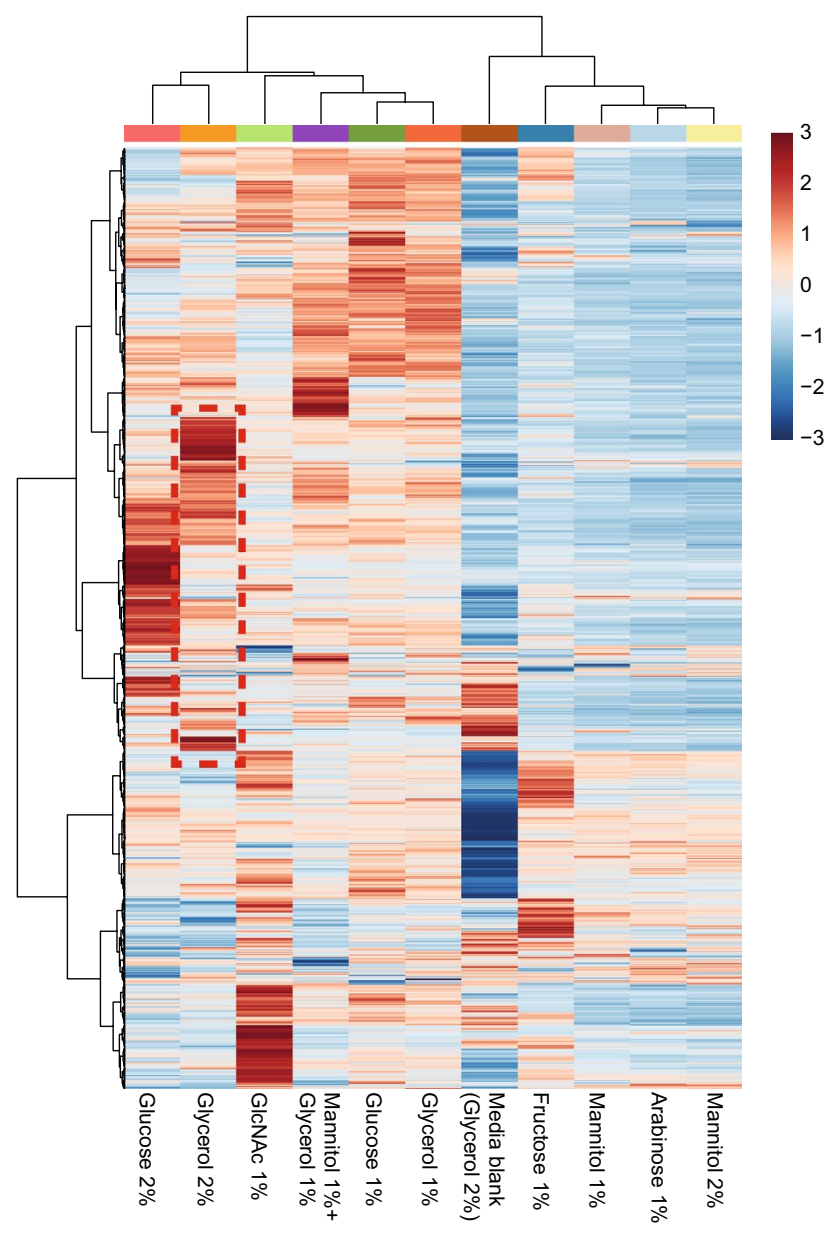

Fig. 1 Analysis of secondary metabolites produced by Streptomyces sp. MBT27. The heat map depicts the relative abundance of the metabolites (rows) produced under different growth conditions (columns) glycerol or glucose from 1 to $2 \%$ had a profound effect on the metabolic profile. Thin layer chromatography (TLC) was conducted to compare metabolic profiles of the $1 \%$ and $2 \%$ glycerol-grown cultures. Interestingly, this revealed that several fluorescent compounds were differentially produced in the extracts of $2 \%$ glycerol-grown cultures relative to those produced in $1 \%$ glycerol (Fig. 2a). In order to provide statistical relevance to the data, the metabolic profiles of $1 \%$ and $2 \%$ glycerol-grown cultures were compared using a volcano plot (Fig. 2b). The volcano plot was then searched for the mass features which increased in production in $2 \%$ glycerol as compared to $1 \%$. A mass of $m / z 281.1151$ (1) stood out as its intensity had increased by around 7000 fold ( $p$ value $=0.002$ ) in cultures fermented in $2 \%$ glycerol as compared to $1 \%$.

Global Natural Product Social (GNPS) molecular networking [45] was employed to detect the MS/MS structural relatedness among molecules in an automated manner; the software generates a molecular network wherein molecules with related scaffolds cluster together [45]. A network representing the ions detected in the crude extract of Streptomyces sp. MBT27 grown with $2 \%$ glycerol was constructed, revealing 183 nodes clustered in 19 spectral families (Fig. 3). GNPS dereplication based on matching with its MS/MS spectral database highlighted some known metabolites. These included anthranilic acid (3), anthranilamide (4) [37], actinomycin D (7), and actinomycin X2 (8) [44]. The annotation of the compounds was supported by comparison of the exact mass, fragmentation pattern, and UV spectra, with reference data. Moreover, crude extracts of MBT27 possessed antimicrobial activity against $B$. subtilis, which was most likely due to the expression of actinomycins (data not shown). Additional metabolites could be dereplicated through manual comparison of their spectral data against the microbial natural products database Antibase. These previously described metabolites were 4(3H)-quinazolinone (5) [23] and 2,2-dimethyl-1,2-dihydroquinazolin-4(3H)-one (6) [8].

Besides known molecules, the network also contained many mass features that could not be assigned to any of the previously identified metabolites. One of these was an ion with an $\mathrm{m} / \mathrm{z}$ value of 281.1151 (1), which was very highly increased in intensity ( 7000 -fold, $p$ value $=0.002$ ) when the glycerol concentration was increased from 1 to $2 \%$. It was closely connected (cosine score 0.91 ) to another upregulated ion with an $\mathrm{m} / \mathrm{z}$ value of 209.0915 (2), which was also not previously reported. Both ions were part of the large spectral family comprising the annotated anthranilic acid (3), anthranilamide (4), and the 4-quinazolinones 5 and $\mathbf{6}$, suggesting that they are structurally related metabolites. It is important to note that the concentrations of quinazolinones $\mathrm{A}$ and $\mathrm{B}$ did not change significantly when the concentration of either mannitol or glucose was doubled from 1 to $2 \%$, or when 

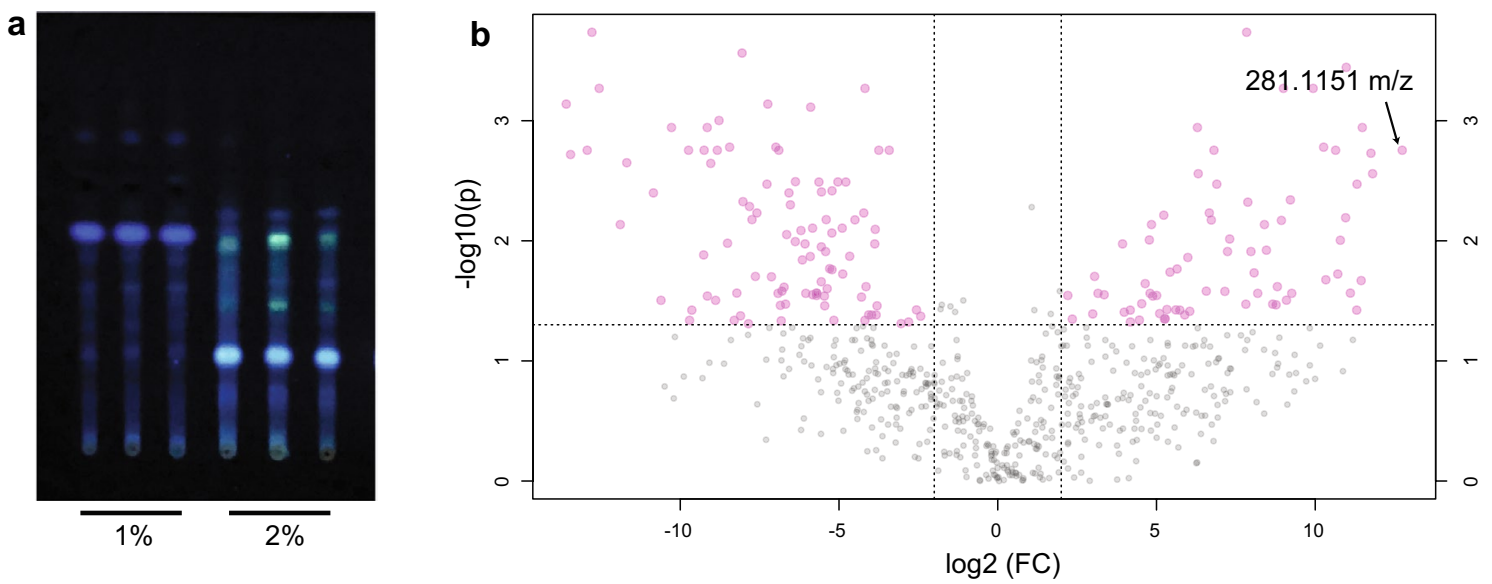

Fig. 2 Secondary metabolite profiles found in 1\% and 2\% glycerolgrown cultures of Streptomyces sp. MBT27. a Thin-layer chromatography of extracts from the cultures grown in MM with either $1 \%$ or $2 \%$ glycerol as the carbon source. b Volcano plot highlighting the differences in metabolite profiles. The $x$ and $y$ axes of the volcano plot represent the $\log 2$ fold changes and the corresponding $-\log 10$ FDR-adjusted $p$-value of all metabolites, respectively. Pink circles represent metabolites with an intensity difference of more than fourfold ( $p$ value $\leq 0.05$ ). Ions present in the left and right quadrants are associated with the $1 \%$ and $2 \%$ glycerol-grown cultures, respectively. Metabolites situated towards the left and right top quadrants represent values of large magnitude fold changes as well as high statistical significance

The relationship between the increase in glycerol con-

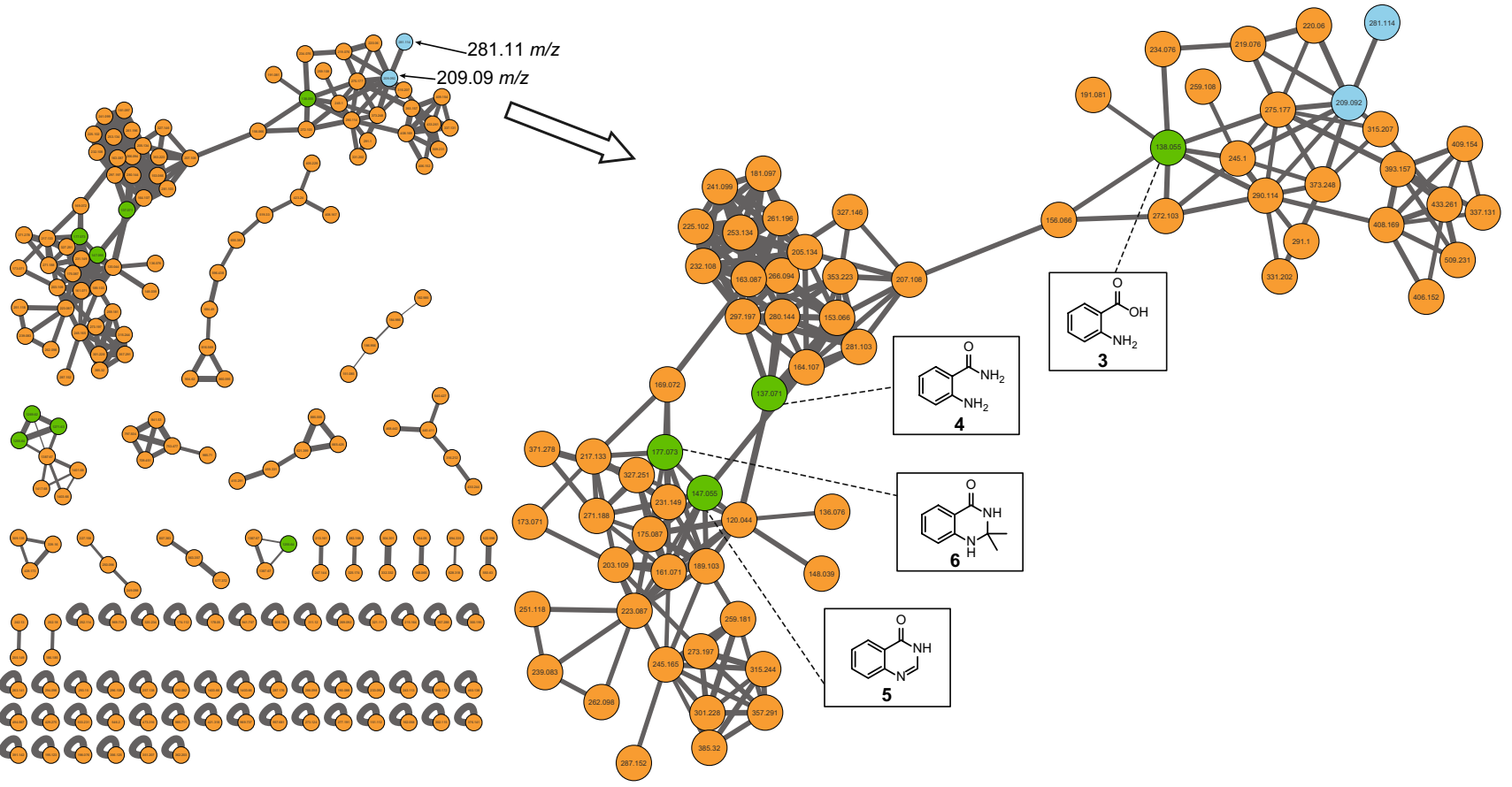

Fig. 3 GNPS molecular network of the ions detected in the crude extract of Streptomyces sp. MBT27 grown in MM with 2\% glycerol. Orange nodes represent all the ions detected in the extract. Green

(Fig. S1). This strongly suggests that the effect of glycerol is not due to increased availability of the carbon source. nodes represent the dereplicated metabolites, while blue nodes represent the novel molecules $\mathbf{1}$ and $\mathbf{2}$ that were upregulated when glycerol concentration was increased from 1 to $2 \%$

centration and the strong increase in the production of the compounds $\mathbf{1}$ and $\mathbf{2}$, was investigated further by expanding the range of glycerol concentrations and analyzing the 
metabolic profiles. For this, Streptomyces sp. MBT27 was cultured in glycerol concentrations ranging from 1 to $4 \%$ (w/v). ANOVA, followed by a post hoc Tukey's HSD test, was performed to trace the variation in the production of $\mathbf{1}$ and 2 among the different culturing conditions. A box plot was used to visualize such variation (Fig. S2). As observed earlier, the production of $\mathbf{1}$ and $\mathbf{2}$ was significantly increased when the glycerol concentration was increased from 1 to $2 \%$. However, further increase in glycerol concentration $(3 \%$ and $4 \%$ ) did not lead to any significant increase in the production of $\mathbf{1}$ and 2. Accordingly, MM with 2\% glycerol was used to culture the bacteria, for the purpose of purification and identification of the new metabolites $\mathbf{1}$ and $\mathbf{2}$.

\section{Isolation and structure elucidation of novel quinazolinones}

To elucidate the structure of $\mathbf{1}$ and $\mathbf{2}$, large-scale fermentation was done to obtain larger quantities of the compounds. For this, Streptomyces sp. MBT27 was fermented in a total of $4 \mathrm{~L}$ of liquid MM supplemented with $2 \%$ glycerol, and the supernatant was extracted with EtOAc. Following repeated chromatographic isolation, compounds $\mathbf{1}$ and $\mathbf{2}$ were obtained as pure, colorless, amorphous powders. Both compounds were fluorescent, showing UV absorption maxima at 224 and $322 \mathrm{~nm}$ and at 226 and $347 \mathrm{~nm}$, respectively. The final structures of $\mathbf{1}$ and $\mathbf{2}$ were determined by the combination of NMR and high-resolution MS (Fig. 4).

The high-resolution mass of $\mathrm{m} / \mathrm{z} 281.1130$ for an $[\mathrm{M}+\mathrm{H}]^{+}$established a molecular formula of $\mathrm{C}_{13} \mathrm{H}_{16} \mathrm{~N}_{2} \mathrm{O}_{5}$, with seven degrees of unsaturation, for 1 (yield $0.25 \mathrm{mg} / \mathrm{L}$ ). The deduced molecular formula was corroborated by the ${ }^{13} \mathrm{C}$ NMR attached proton test (APT) spectrum that exhibited 13 carbons in total. On the other hand, the ${ }^{1} \mathrm{H}$ NMR spectrum of 1 (Table 1) presented four coupling aromatic signals at $\delta_{\mathrm{H}} 7.88(\mathrm{dd}, J=7.8,1.2, \mathrm{H}-5), 7.07$ (t, $\left.J=7.8, \mathrm{H}-6\right), 7.46$ (td, $J=7.8,1.2, \mathrm{H}-7$ ), and 7.23 (brd, $J=7.8, \mathrm{H}-8$ ), indicating an $o$-disubstituted aromatic benzene system. The HMBC correlation (Fig. 5) from H-5 to C-4 $\left(\delta_{\mathrm{C}} 166.1\right)$ confirmed one of substituents on the benzene ring to be an ester/amide carbonyl group, while the downfield chemical shift of C-8a at $\delta_{\mathrm{C}} 143.7$ indicated it was nitrogenated. Four O-bearing

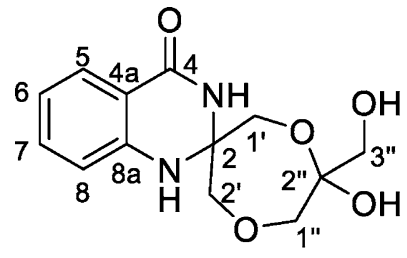

Quinazolinone A (1)

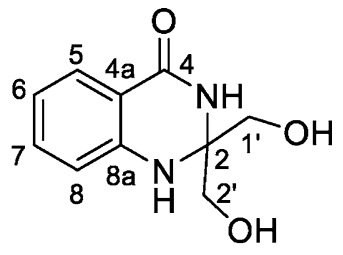

Quinazolinone B (2)
Fig. 4 Chemical structures of quinazolinones A (1) and B (2)
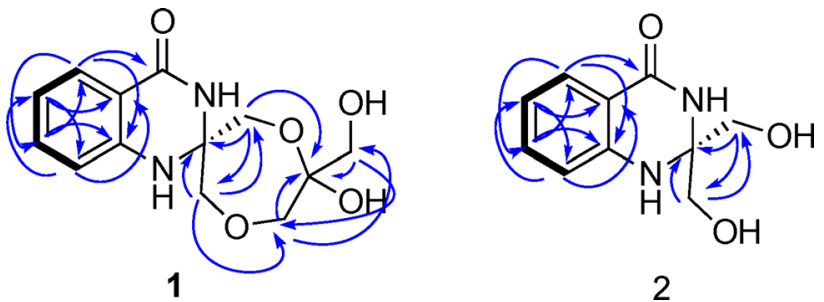

Fig. 5 Key COSY () and HMBC () correlations for $\mathbf{1}$ and $\mathbf{2}$

methylene groups at $\delta_{\mathrm{H}} 4.43\left(\mathrm{~d}, J=9.0, \mathrm{H}-1^{\prime} \mathrm{a}\right)$ and $4.24(\mathrm{~d}$, $\left.J=9.0, \mathrm{H}-1^{\prime} \mathrm{b}\right) ; 3.59$ (d, $\left.J=11.4, \mathrm{H}-2^{\prime} \mathrm{a}\right)$ and 3.47 (d, $J=11.4$, H-2'b); 3.86 (d, $\left.J=12.6, \mathrm{H}-1^{\prime \prime} \mathrm{a}\right)$ and 3.80 (d, $J=12.6$, $\mathrm{H}-1 " \mathrm{~b})$; and $3.66\left(\mathrm{~d}, J=3.0, \mathrm{H}_{2}-3^{\prime \prime}\right)$ were resolved by HSQC experiments. Three of these methylene groups were part of a 1,4-dioxepane ring system, which was established based on the HMBC correlations observed from $\mathrm{H}-1^{\prime}$ to $\mathrm{C}-2\left(\delta_{\mathrm{C}}\right.$ 79.0) and $\mathrm{C}-2^{\prime}\left(\delta_{\mathrm{C}} 64.6\right)$, from $\mathrm{H}-2^{\prime}$ to $\mathrm{C}-1^{\prime \prime}\left(\delta_{\mathrm{C}} 65.7\right)$, and from $\mathrm{H}-1$ ' to the hemiacetal $\mathrm{C}-2^{\prime \prime}\left(\delta_{\mathrm{C}} 102.9\right)$. The remaining oxymethylene group $\mathrm{CH}_{2}-3$ " was connected to $\mathrm{C}-2$ " based on the HMBC correlations observed from $\mathrm{H}_{2}-3$ " to C-1" and C-2". The two substructures obtained accounted for all of the oxygens and six out of the seven degrees of unsaturation required by the molecular formula of $\mathbf{1}$. Accordingly, an additional ring including two nitrogen atoms was deduced to connect the two substructures, forming a 4-quinazolinone ring. Consequently, compound $\mathbf{1}$ was identified to be a $d i$ glycerolated 4-quinozolinone, and was named quinazolinone A.

The $[\mathrm{M}+\mathrm{H}]^{+}$molecular ion at $m / z 209.0932$ in the ESI-HRMS spectrum resulted in a molecular formula of $\mathrm{C}_{10} \mathrm{H}_{12} \mathrm{~N}_{2} \mathrm{O}_{3}$ for 2 (yield $0.3 \mathrm{mg} / \mathrm{L}$ ). The ${ }^{13} \mathrm{C}$ NMR spectrum presented 10 signals, which was consistent with the molecular formula (Table 1). The ${ }^{1} \mathrm{H}$ NMR spectrum of 2 resembled that of $\mathbf{1}$ in the downfield aromatic region, and the major difference is the absence of a set of signals for the additional glycerol unit. Further HMBC experiment confirmed $\mathbf{2}$ is a mono-glycerolated 4-quinazolinone (Fig. 5). Compound 2 was named quinazolinone $\mathrm{B}$.

The 4-quinazoline alkaloids represent an important class of nitrogen-containing heterocyclic compounds [27], which have highly diverse biological activities, such as antitumor, anti-inflammatory, antihypertensive, antimicrobial, anticonvulsant and antifungal activities [18]. Though the basic bicyclic core (the fused benzene ring and pyrimidine ring) is normally conserved in most of naturally occurring 4-quinazoline derivatives [27], a variety of structural substitutions have oftentimes been found on the pyrimidine ring of the 4-quinazolinone. The chemical structures of quinazolinones $\mathrm{A}$ and $\mathrm{B}$ and the glycerol-dependent production suggest that glycerol participates in the construction of the ring system. We propose that quinazolinones A and B form a new 


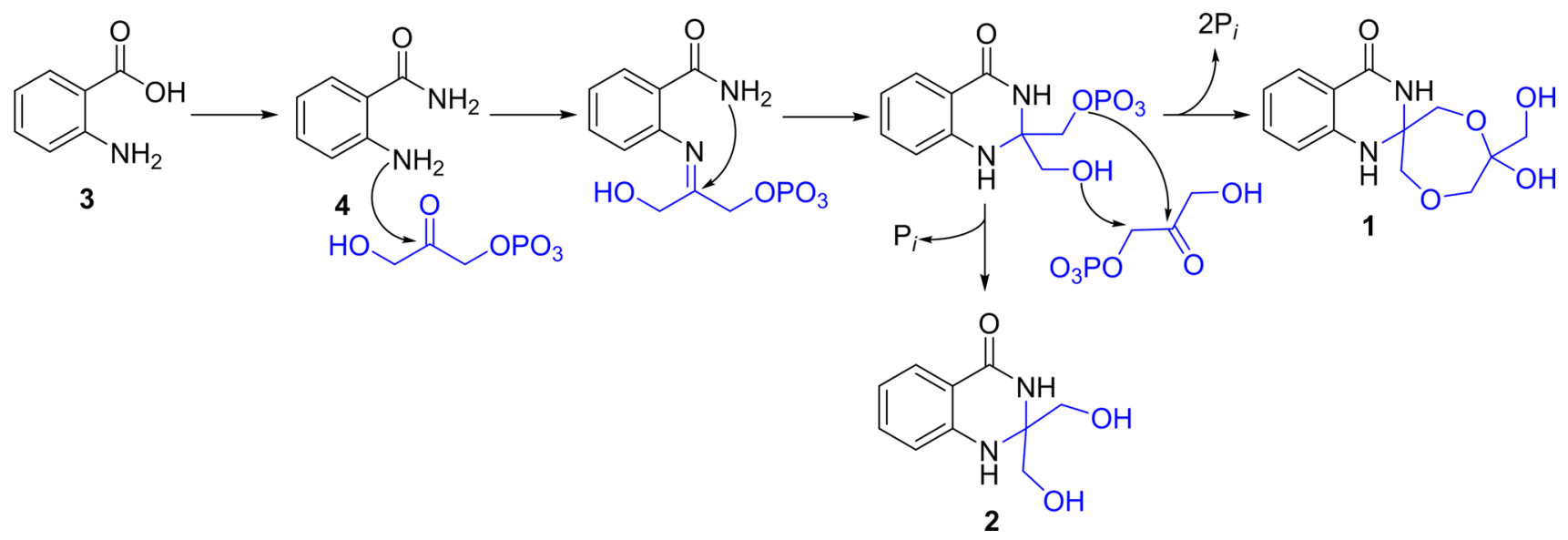

Fig. 6 Proposed biosynthetic pathway for quinazolinone A (1) and B (2)

sub-branch in the family of the quinazolines. Particularly, quinazolinone A contains an exciting seven-membered ring that we believe may be formed by intermolecular etherification of two units of glycerol, and this ring is further linked with the quinazolinone backbone by a spiro atom at $\mathrm{C}-2$, which is unprecedented. Antimicrobial activities of compounds $\mathbf{1}$ and $\mathbf{2}$ were tested against $B$. subtilis and $E$. coli. None of the compounds showed antimicrobial activity against these indictor strains (data not shown).

\section{Biosynthesis of quinazolinones A and B}

In our study, the production of $\mathbf{1}$ and $\mathbf{2}$ was dramatically enhanced in the cultures with $2 \%$ glycerol. Elicitation by glycerol is an indication that glycerol may play a key role during their biosynthesis. The aromatic ring system of quinazoline alkaloids is known to be derived from anthranilic acid, which in turn is biosynthesized through the shikimate pathway [12]. Detection of anthranilamide in the extract of Streptomyces sp. MBT27 leads to the assumption that the biosynthesis of the isolated quinazolinones starts from anthranilic acid, which is then converted to anthranilamide (Fig. 6). Further successive attachments of two molecules of glycerol then results in the formation of $\mathbf{2}$, followed by $\mathbf{1}$. However, multiple biosynthetic routes have been proposed for quinazolinones, because the $\mathrm{C}-2$ residue of the quinazoline ring may originate from various precursors. For example, the C-2 and the remaining non-aromatic part of the quinazolinone alkaloid chrysogine, produced by Penicillium chrysogenum, are biosynthesized from pyruvic acid via an NRPS system [47], while the non-aromatic part of the quinazoline alkaloid peganine, produced by the plant Peganum harmala, is derived from ornithine [12]. Furthermore, fumiquinazoline $\mathrm{F}$ originates from a fungal nonribosomal peptide synthetase (TqaA) and is biosynthesized from anthranilic acid, L-tryptophan and L-alanine [14].

In conclusion, the metabolic potential of Streptomyces sp. MBT27 was analyzed under different nutritional conditions, showing major changes in the metabolome, depending on the carbon source used. In particular, the relatively unspectacular change from $1 \%$ to $2 \%$ glycerol resulted in a surprisingly global change in the secondary metabolome, with some compounds changing by almost four orders of magnitude. The use of GNPS molecular networking, together with LC-MS based dereplication, allowed us to annotate a cluster of quinazolinone-family molecules that connects to anthranilic acid and anthranilamide. Isolation and structure elucidation revealed the novel alkaloid natural products quinazolinone A and B. Based on the structures, the biosynthesis of the compounds most likely involves the conversion of anthranilic acid to anthranilamide, which is subsequently attached to two glycerol units, to produce compounds $\mathbf{1}$ and 2. Identification of the biosynthetic gene cluster and subsequent analysis of the individual enzymatic reactions should reveal the precise biosynthetic pathway for these exciting novel molecules.

Open Access This article is distributed under the terms of the Creative Commons Attribution 4.0 International License (http://creativeco mmons.org/licenses/by/4.0/), which permits unrestricted use, distribution, and reproduction in any medium, provided you give appropriate credit to the original author(s) and the source, provide a link to the Creative Commons license, and indicate if changes were made.

\section{References}

1. Baltz RH (2008) Renaissance in antibacterial discovery from actinomycetes. Curr Opin Pharmacol 8:557-563

2. Barka EA, Vatsa P, Sanchez L, Gavaut-Vaillant N, Jacquard C, Meier-Kolthoff J, Klenk HP, Clément C, Oudouch Y, van Wezel 
GP (2016) Taxonomy, physiology, and natural products of the Actinobacteria. Microbiol Mol Biol Rev 80:1-43

3. Bentley SD, Chater KF, Cerdeno-Tarraga AM, Challis GL, Thomson NR, James KD, Harris DE, Quail MA, Kieser H, Harper D, Bateman A, Brown S, Chandra G, Chen CW, Collins M, Cronin A, Fraser A, Goble A, Hidalgo J, Hornsby T, Howarth S, Huang CH, Kieser T, Larke L, Murphy L, Oliver K, O’Neil S, Rabbinowitsch E, Rajandream MA, Rutherford K, Rutter S, Seeger K, Saunders D, Sharp S, Squares R, Squares S, Taylor K, Warren T, Wietzorrek A, Woodward J, Barrell BG, Parkhill J, Hopwood DA (2002) Complete genome sequence of the model actinomycete Streptomyces coelicolor A3(2). Nature 417:141-147

4. Bérdy J (2005) Bioactive microbial metabolites. J Antibiot (Tokyo) 58:1-26

5. Bode HB, Bethe B, Höfs R, Zeeck A (2002) Big effects from small changes: possible ways to explore nature's chemical diversity. ChemBioChem 3:619-627

6. Chambers MC, Maclean B, Burke R, Amodei D, Ruderman DL, Neumann S, Gatto L, Fischer B, Pratt B, Egertson J, Hoff K, Kessner D, Tasman N, Shulman N, Frewen B, Baker TA, Brusniak MY, Paulse C, Creasy D, Flashner L, Kani K, Moulding C, Seymour SL, Nuwaysir LM, Lefebvre B, Kuhlmann F, Roark J, Rainer P, Detlev S, Hemenway T, Huhmer A, Langridge J, Connolly B, Chadick T, Holly K, Eckels J, Deutsch EW, Moritz RL, Katz JE, Agus DB, MacCoss M, Tabb DL, Mallick P (2012) A cross-platform toolkit for mass spectrometry and proteomics. Nat Biotechnol 30:918-920

7. Chong J, Soufan O, Li C, Caraus I, Li S, Bourque G, Wishart DS, Xia J (2018) MetaboAnalyst 4.0: towards more transparent and integrative metabolomics analysis. Nucleic Acids Res 46:W486-W494

8. Conti R, Chagas FO, Caraballo-Rodriguez AM, Melo WG, do Nascimento AM, Cavalcanti BC, de Moraes MO, Pessoa C, Costa-Lotufo LV, Krogh R, Andricopulo AD, Lopes NP, Pupo MT (2016) Endophytic actinobacteria from the brazilian medicinal plant Lychnophora ericoides Mart. and the biological potential of their secondary metabolites. Chem Biodivers 13:727-736

9. Cooper MA, Shlaes D (2011) Fix the antibiotics pipeline. Nature 472:32

10. Craney A, Ozimok C, Pimentel-Elardo SM, Capretta A, Nodwell JR (2012) Chemical perturbation of secondary metabolism demonstrates important links to primary metabolism. Chem Biol 19:1020-1027

11. Cruz-Morales P, Vijgenboom E, Iruegas-Bocardo F, Girard G, Yanez-Guerra LA, Ramos-Aboites HE, Pernodet JL, Anne J, van Wezel GP, Barona-Gomez F (2013) The genome sequence of Streptomyces lividans 66 reveals a novel tRNA-dependent peptide biosynthetic system within a metal-related genomic island. Genome Biol Evol 5:1165-1175

12. Dewick PM (2009) Medicinal natural products: a biosynthetic approach, 3rd edn. Ohn Wiley \& Sons, Chichester

13. Du C, van Wezel GP (2018) Mining for microbial gems: integrating proteomics in the postgenomics natural product discovery pipeline. Proteomics 18:1700332

14. Gao X, Chooi YH, Ames BD, Wang P, Walsh CT, Tang Y (2011) Fungal indole alkaloid biosynthesis: genetic and biochemical investigation of the tryptoquialanine pathway in Penicillium aethiopicum. J Am Chem Soc 133:2729-2741

15. Gaudencio SP, Pereira F (2015) Dereplication: racing to speed up the natural products discovery process. Nat Prod Rep 32:779-810

16. Gubbens J, Zhu H, Girard G, Song L, Florea BI, Aston P, Ichinose K, Filippov DV, Choi YH, Overkleeft HS, Challis GL, van Wezel GP (2014) Natural product proteomining, a quantitative proteomics platform, allows rapid discovery of biosynthetic gene clusters for different classes of natural products. Chem Biol 21:707-718
17. Hameed A, Al-Rashida M, Uroos M, Ali SA, Arshia Ishtiaq M, Khan KM (2018) Quinazoline and quinazolinone as important medicinal scaffolds: a comparative patent review (2011-2016). Expert Opin Ther Pat 28:281-297

18. He D, Wang M, Zhao S, Shu Y, Zeng H, Xiao C, Lu C, Liu Y (2017) Pharmaceutical prospects of naturally occurring quinazolinone and its derivatives. Fitoterapia 119:136-149

19. Ikeda H, Ishikawa J, Hanamoto A, Shinose M, Kikuchi H, Shiba T, Sakaki Y, Hattori M, Omura S (2003) Complete genome sequence and comparative analysis of the industrial microorganism Streptomyces avermitilis. Nat Biotechnol 21:526-531

20. Jafari E, Khajouei MR, Hassanzadeh F, Hakimelahi GH, Khodarahmi GA (2016) Quinazolinone and quinazoline derivatives: recent structures with potent antimicrobial and cytotoxic activities. Res Pharm Sci 11:1-14

21. Kieser T, Bibb MJ, Buttner MJ, Chater KF, Hopwood DA (2000) Practical Streptomyces genetics. John Innes Foundation, Norwich

22. Kolter R, van Wezel GP (2016) Goodbye to brute force in antibiotic discovery? Nat Microbiol 1:15020

23. Kornsakulkarn J, Saepua S, Srijomthong K, Rachtawee P, Thongpanchang C (2015) Quinazolinone alkaloids from actinomycete Streptomyces sp. BCC 21795. Phytochem Lett 12:6-8

24. Kshirsagar UA (2015) Recent developments in the chemistry of quinazolinone alkaloids. Org Biomol Chem 13:9336-9352

25. Laatsch H (2012) Antibase 2012, the natural compound Identifier. Wiley-VCH Weinheim, Germany

26. Martin JF, Sola-Landa A, Santos-Beneit F, Fernandez-Martinez LT, Prieto C, Rodriguez-Garcia A (2011) Cross-talk of global nutritional regulators in the control of primary and secondary metabolism in Streptomyces. Microb Biotechnol 4:165-174

27. Michael JP (2000) Quinoline, quinazoline and acridone alkaloids. Nat Prod Rep 17:603-620

28. Nett M, Ikeda H, Moore BS (2009) Genomic basis for natural product biosynthetic diversity in the actinomycetes. Nat Prod Rep 26:1362-1384

29. Payne DJ, Gwynn MN, Holmes DJ, Pompliano DL (2007) Drugs for bad bugs: confronting the challenges of antibacterial discovery. Nat Rev Drug Discov 6:29-40

30. Pluskal T, Castillo S, Villar-Briones A, Orešič M (2010) MZmine 2: modular framework for processing, visualizing, and analyzing mass spectrometry-based molecular profile data. BMC Bioinformatics 11:395

31. Rice LB (2008) Federal funding for the study of antimicrobial resistance in nosocomial pathogens: no ESKAPE. J Infect Dis 197:1079-1081

32. Rigali S, Titgemeyer F, Barends S, Mulder S, Thomae AW, Hopwood DA, van Wezel GP (2008) Feast or famine: the global regulator DasR links nutrient stress to antibiotic production by Streptomyces. EMBO Rep 9:670-675

33. Romano S, Jackson SA, Patry S, Dobson ADW (2018) Extending the "one strain many compounds" (OSMAC) principle to marine microorganisms. Mar Drugs 16:244

34. Rutledge PJ, Challis GL (2015) Discovery of microbial natural products by activation of silent biosynthetic gene clusters. Nat Rev Microbiol 13:509-523

35. Sanchez S, Chavez A, Forero A, Garcia-Huante Y, Romero A, Sanchez M, Rocha D, Sanchez B, Avalos M, Guzman-Trampe S, Rodriguez-Sanoja R, Langley E, Ruiz B (2010) Carbon source regulation of antibiotic production. J Antibiot (Tokyo) 63:442-459

36. Schulze CJ, Bray WM, Woerhmann MH, Stuart J, Lokey RS, Linington RG (2013) "Function-first" lead discovery: mode of action profiling of natural product libraries using image-based screening. Chem Biol 20:285-295

37. Shaaban KA, Shepherd MD, Ahmed TA, Nybo SE, Leggas M, Rohr J (2012) Pyramidamycins A-D and 
3-hydroxyquinoline-2-carboxamide; cytotoxic benzamides from Streptomyces sp. DGC1. J Antibiot (Tokyo) 65:615-622

38. Shannon P, Markiel A, Ozier O, Baliga NS, Wang JT, Ramage D, Amin N, Schwikowski B, Ideker T (2003) Cytoscape: a software environment for integrated models of biomolecular interaction networks. Genome Res 13:2498-2504

39. Swiatek-Polatynska MA, Bucca G, Laing E, Gubbens J, Titgemeyer F, Smith CP, Rigali S, van Wezel GP (2015) Genomewide analysis of In vivo binding of the master regulator DasR in Streptomyces coelicolor identifies novel non-canonical targets. PLoS One 10:e0122479

40. Urem M, Swiatek-Polatynska MA, Rigali S, van Wezel GP (2016) Intertwining nutrient-sensory networks and the control of antibiotic production in Streptomyces. Mol Microbiol 102:183-195

41. van der Heul HU, Bilyk BL, McDowall KJ, Seipke RF, van Wezel GP (2018) Regulation of antibiotic production in Actinobacteria: new perspectives from the post-genomic era. Nat Prod Rep 35:575-604

42. van der Meij A, Worsley SF, Hutchings MI, van Wezel GP (2017) Chemical ecology of antibiotic production by actinomycetes. FEMS Microbiol Rev 41:392-416

43. van Wezel GP, McKenzie NL, Nodwell JR (2009) Applying the genetics of secondary metabolism in model actinomycetes to the discovery of new antibiotics. Methods Enzymol 458:117-141

44. Vater J, Crnovcic I, Semsary S, Keller U (2014) MALDI-TOF mass spectrometry, an efficient technique for in situ detection and characterization of actinomycins. J Mass Spectrom 49:210-222

45. Wang M, Carver JJ, Phelan VV, Sanchez LM, Garg N, Peng Y, Nguyen DD, Watrous J, Kapono CA, Luzzatto-Knaan T, Porto C, Bouslimani A, Melnik AV, Meehan MJ, Liu WT, Crusemann M, Boudreau PD, Esquenazi E, Sandoval-Calderon M, Kersten RD, Pace LA, Quinn RA, Duncan KR, Hsu CC, Floros DJ, Gavilan RG, Kleigrewe K, Northen T, Dutton RJ, Parrot D, Carlson EE, Aigle B, Michelsen CF, Jelsbak L, Sohlenkamp C, Pevzner P, Edlund A, McLean J, Piel J, Murphy BT, Gerwick L, Liaw CC, Yang YL, Humpf HU, Maansson M, Keyzers RA, Sims AC, Johnson AR, Sidebottom AM, Sedio BE, Klitgaard A, Larson CB, Boya PC, Torres-Mendoza D, Gonzalez DJ, Silva DB, Marques LM, Demarque DP, Pociute E, O'Neill EC, Briand E, Helfrich EJ, Granatosky EA, Glukhov E, Ryffel F, Houson H, Mohimani H, Kharbush JJ, Zeng Y, Vorholt JA, Kurita KL, Charusanti P, McPhail KL, Nielsen KF, Vuong L, Elfeki M, Traxler MF, Engene N, Koyama N, Vining OB, Baric R, Silva RR, Mascuch SJ, Tomasi S, Jenkins S, Macherla V, Hoffman T, Agarwal V, Williams PG, Dai J, Neupane R, Gurr J, Rodriguez AM, Lamsa A, Zhang C, Dorrestein K, Duggan BM, Almaliti J, Allard PM, Phapale P, Nothias LF, Alexandrov T, Litaudon M, Wolfender JL, Kyle JE, Metz TO, Peryea T, Nguyen DT, VanLeer D, Shinn P, Jadhav A, Muller R, Waters KM, Shi W, Liu X, Zhang L, Knight R, Jensen PR, Palsson BO, Pogliano K, Linington RG, Gutierrez M, Lopes NP, Gerwick WH, Moore BS, Dorrestein PC, Bandeira
N (2016) Sharing and community curation of mass spectrometry data with Global Natural Products Social Molecular Networking. Nat Biotechnol 34:828-837

46. Winnikoff JR, Glukhov E, Watrous J, Dorrestein PC, Gerwick WH (2014) Quantitative molecular networking to profile marine cyanobacterial metabolomes. J Antibiot (Tokyo) 67:105-112

47. Wollenberg RD, Saei W, Westphal KR, Klitgaard CS, Nielsen KL, Lysoe E, Gardiner DM, Wimmer R, Sondergaard TE, Sorensen JL (2017) Chrysogine biosynthesis is mediated by a two-module nonribosomal peptide synthetase. J Nat Prod 80:2131-2135

48. Wu C, Choi YH, van Wezel GP (2016) Metabolic profiling as a tool for prioritizing antimicrobial compounds. J Ind Microbiol Biotechnol 43:299-312

49. Wu C, Du C, Gubbens J, Choi YH, van Wezel GP (2015) Metabolomics-driven discovery of a prenylated isatin antibiotic produced by Streptomyces species MBT28. J Nat Prod 78:2355-2363

50. Wu C, Kim HK, van Wezel GP, Choi YH (2015) Metabolomics in the natural products field - a gateway to novel antibiotics. Drug Discov Today Technol 13:11-17

51. Wu C, Medema MH, Lakamp RM, Zhang L, Dorrestein PC, Choi YH, van Wezel GP (2016) Leucanicidin and endophenasides result from methyl-rhamnosylation by the same tailoring enzymes in Kitasatospora sp. MBT66. ACS Chem Biol 11:478-490

52. Wu C, Zacchetti B, Ram AF, van Wezel GP, Claessen D, Hae Choi $Y$ (2015) Expanding the chemical space for natural products by Aspergillus-Streptomyces co-cultivation and biotransformation. Sci Rep 5:10868

53. Wu C, Zhu H, van Wezel GP, Choi YH (2016) Metabolomicsguided analysis of isocoumarin production by Streptomyces species MBT76 and biotransformation of flavonoids and phenylpropanoids. Metabolomics 12:90

54. Yang JY, Sanchez LM, Rath CM, Liu X, Boudreau PD, Bruns N, Glukhov E, Wodtke A, de Felicio R, Fenner A, Wong WR, Linington RG, Zhang L, Debonsi HM, Gerwick WH, Dorrestein PC (2013) Molecular networking as a dereplication strategy. J Nat Prod 76:1686-1699

55. Yoon V, Nodwell JR (2014) Activating secondary metabolism with stress and chemicals. J Ind Microbiol Biotechnol 41:415-424

56. Zhu H, Sandiford SK, van Wezel GP (2014) Triggers and cues that activate antibiotic production by actinomycetes. J Ind Microbiol Biotechnol 41:371-386

57. Zhu H, Swierstra J, Wu C, Girard G, Choi YH, van Wamel W, Sandiford SK, van Wezel GP (2014) Eliciting antibiotics active against the ESKAPE pathogens in a collection of actinomycetes isolated from mountain soils. Microbiology 160:1714-1725

Publisher's Note Springer Nature remains neutral with regard to jurisdictional claims in published maps and institutional affiliations. 